\title{
Temperature-dependent current-voltage characteristics of Pd/ZnO Schottky barrier diodes and the determination of the Richardson constant
}

\author{
M.A. Mayimele, M. Diale*, W. Mtangi, F.D. Auret \\ Physics department, University of Pretoria, Pretoria 0002, South Africa
}

\begin{abstract}
We report on a systematic investigation of temperature dependent current-voltage $(I-V)$ characteristics of $\mathrm{Pd} / \mathrm{ZnO}$ Schottky barrier diodes in the 30-300 K temperature range. The ideality factor was observed to decrease with increase in temperature, whilst the barrier height increases with increase in temperature. The observed trend has been attributed to barrier inhomogeneities, which results in a distribution of barrier heights at the interface. Using the dependence of saturation current values on temperature, we have calculated the Richardson constant $\left(A^{*}\right)$ which was investigated in the two distinct temperature regions: $140-200 \mathrm{~K}$ and $210-300 \mathrm{~K}$ and values of $3 \times 10^{-12}$ and $3 \times 10^{-9} \mathrm{~A} \mathrm{~cm}^{-2} \mathrm{~K}^{-2}$ were obtained, respectively. A mean barrier height of $0.97 \mathrm{eV}$ was obtained in the $140-300 \mathrm{~K}$ temperature range. Applying the barrier height inhomogeneities correction, the value of $A^{*}$ was obtained from the modified Richardson plots as 39.43 and $39.03 \mathrm{~A} \mathrm{~cm}^{-2} \mathrm{~K}^{-2}$ in the $140-200 \mathrm{~K}$ and 210-300 K temperature range. The modified Richardson constant $\left(A^{* *}\right)$ has proved to be strongly affected by barrier inhomogeneities and dependent on contact quality.
\end{abstract}

Keywords: $I$ - $V$ characteristics, temperature-dependent, Richardson constant, Barrier Inhomogeneities, Gaussian distribution.

Corresponding author; M. Diale; email: mmantsae.diale@ up.ac.za; Tel:+27124204418 


\section{Introduction}

Zinc Oxide $(\mathrm{ZnO})$ has gained a lot of global interest in the research community because of its direct and wide band gap of $3.37 \mathrm{eV}$. It has a larger exciton binding energy of $60 \mathrm{meV}$ and hence a high efficiency in excitonic emission [1, 2]. In addition, it has superior electronic properties such as high breakdown voltage, high electron saturation velocity, high thermal conductivity and is very resistant to high-energy radiation, making it a very suitable candidate for applications in space and around nuclear reactors [3, 4]. Furthermore, $\mathrm{ZnO}$ has found applications in a number of semiconductor electronic devices such as ultraviolet and bluerange optoelectronics, lasers, photovoltaics, field effect transistors, microwave diodes and sensors [5]. Another attractive characteristic of $\mathrm{ZnO}$ is its amenability to conventional wet chemistry etching, being compatible with silicon technology. The ease of etching $\mathrm{ZnO}$ with all acids and alkalis offers an opportunity for the fabrication of small-size devices [4, 6]. In realizing semiconductor-based devices, high quality Schottky contacts are critical. The fabrication of semiconductor-based devices is of vital importance as it determines the use of the material in both electronic and opto-electronic applications [4]. The fabrication of high quality devices is influenced by several factors, some of which include, but not limited to semiconductor surface contaminants, metal-surface chemical reaction and subsurface defects $[1,7,8]$. These factors can in turn influence the current-voltage characteristics of devices such as current transport mechanisms.

A good understanding of current transport mechanisms is required for a complete explanation of the current transport across a metal-semiconductor (MS) contact. Several researchers have studied current transport properties of Schottky contacts at room temperature in which a thermionic emission (TE) model has been used to calculate the diode parameters [9, 10]. In these calculations, it was assumed that TE is the dominant current transport mechanism. However, it has been noted and reported that the analysis of the electrical characteristics of 
the Schottky barrier diodes (SBDs) only at room temperature does not give sufficient information about the conduction process or the barrier formation at the MS interface $[9,11$, 12]. In cases where current-voltage measurements were performed at other temperatures other than room temperature, it has been demonstrated that $\mathrm{TE}$ is not the only transport mechanism [13-16]. An assumption that TE is the only mechanism responsible for the transport of charge carriers across the MS interface could result in erroneous values of the diode parameters. Since transport mechanisms varies in different temperature ranges, there is a need to perform further analysis on the temperature-dependence of $I$ - $V$ measurements to comprehensively describe the current transport mechanisms and obtain accurate values of the diode parameters at different temperatures.

Several researchers $[1,5,17-19]$ have investigated the temperature-dependence of the $I-V$ characteristics of metal/ZnO Schottky diodes and determine several diode parameters in different temperature regions. Asil et al [19] investigated the temperature dependence of current-voltage characteristics on electro-deposited $\mathrm{p}-\mathrm{ZnO} / \mathrm{n}-\mathrm{Si}$ heterojunctions. The authors reported a mean barrier height of $0.818 \mathrm{eV}$ in the 160-300 K temperature range. Gur et al [2] studied high temperature Schottky diode characteristics of bulk $\mathrm{ZnO}$ and reported a mean barrier height of $0.74 \mathrm{eV}$ in the $240-400 \mathrm{~K}$ temperature range. Mtangi et al [20] investigated the temperature dependence of $\mathrm{Pd} / \mathrm{ZnO}$ SBDs and reported a mean barrier height of $0.50 \mathrm{eV}$ in the $180-300 \mathrm{~K}$ temperature range. Kim et al [17] investigated the temperature-dependence of Ag Schottky contacts to differently grown O-polar bulk ZnO. From their $I-V$ characteristics, the authors revealed a mean barrier height of $0.701 \mathrm{eV}$ in the 200-300 K temperature range. Furthermore, Von Wenchstern et al [21] investigated the temperature dependence of the I-V characteristics on Pd Schottky contacts on $\mathrm{ZnO}$ thin films and reported a mean barrier height of $1.16 \pm 0.04 \mathrm{eV}$ in the 200290 K temperature range. Since the researchers obtained different values of barrier heights in 
different temperature ranges, this shows that the barrier height is strongly dependent on temperature and hence the need to conduct further studies on temperature-dependence of $I$ - $V$ measurements in order to determine the accurate values of barrier heights in different temperature regimes.

Since the determination of the barrier height involves the use of the Richardson constant $A^{*}$, there is also a need to know the exact value of the Richardson constant for the temperature range in which the barrier height is being evaluated. Several reports have revealed a variation of $A^{*}$, where different values have been obtained or calculated in different temperature regions $[2,17,20,22,23]$ as indicated in table 1 . These deviations have been explained by assuming the presence of the barrier height inhomogeneities, where two different approaches

\begin{tabular}{llll}
\hline \hline & Richardson Constant & Temparature range & Authors \\
& $\left(\mathrm{A} \mathrm{cm}^{-2} \mathrm{~K}^{-2}\right)$ & $(\mathrm{K})$ & \\
\hline 1 & 0.272 & $300-420$ & {$[26]$} \\
2 & 0.248 & $240-440$ & {$[2]$} \\
3 & 10 & $293-423$ & {$[39]$} \\
4 & 19.54 & $300-423$ & {$[22]$} \\
5 & 46.3 & $100-300$ & {$[17]$} \\
6 & 123 & $210-300$ & {$[23]$} \\
7 & 167 & $80-180$ & {$[20]$} \\
\hline \hline
\end{tabular}

Table 1. Richardson constants determined in different temperature ranges.

were used in defining the inhomogeneities. One approach, assumes a continuous spatial distribution of the Schottky barrier height (SBH) and the total current cross a Schottky diode is simply calculated by integrating the current determined by the ideal TE theory with an indi- 
vidual barrier height and weighted by the distribution function. In another approach, it is assumed that some small patches of low SBH are embedded in the uniform SBH area.

The deviation from the TE model observed in the $I-V$ characteristics could be quantitatively explained by the TE mechanism with a Gaussian distribution of the Barrier heights [24]. Hence, we investigated temperature-dependent $I-V$ measurements on $\mathrm{Pd} / \mathrm{ZnO}$ SBD in the 30-300 K temperature range and determined the Richardson constant that is closer to the theoretical value $\mathrm{n}-\mathrm{ZnO}$ using the modified plot.

\section{Experiment}

In this study, we used bulk single crystals of undoped $\mathrm{ZnO}$ samples from Cermet Inc [25]. Prior to the fabrication of the Schottky and Ohmic contacts, the samples were degreased in acetone, then methanol for five minutes in an ultrasonic bath. The five minutes degreasing in methanol was followed by three minutes boiling in hydrogen peroxide at a temperature of $100^{\circ} \mathrm{C}$. After treatment with hydrogen peroxide, the samples were blown dry with nitrogen gas. Ohmic contacts with composition of $\mathrm{Al} / \mathrm{Au}$ and relative thicknesses of 50/30 $\mathrm{nm}$ as shown in figure 1, were deposited on the $\mathrm{Zn}$ polar face using the resistive evaporation

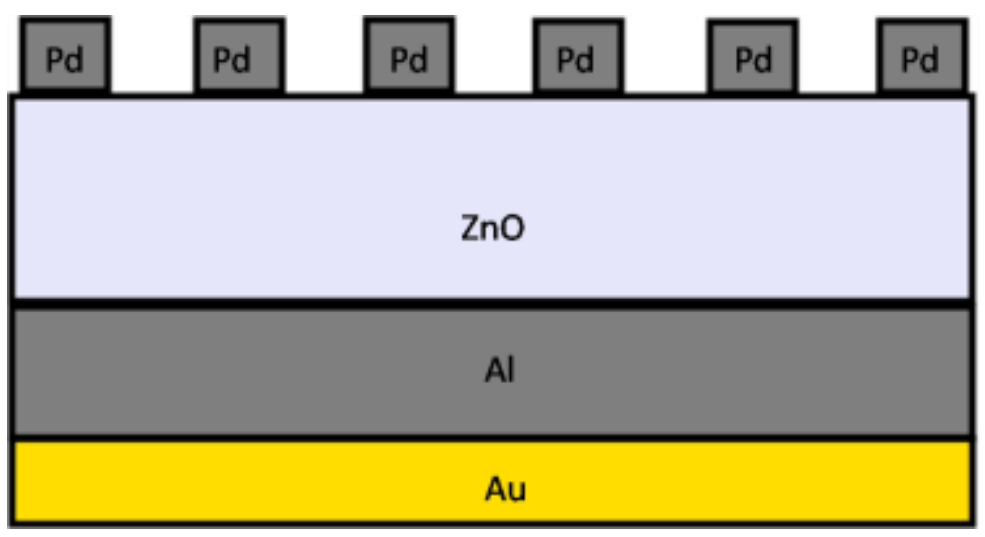

Figure 1. Schematic diagram of $\mathrm{Pd} / \mathrm{ZnO} / \mathrm{Al} / \mathrm{Au} \mathrm{SBD}$.

technique at a pressure of approximately $1 \times 10^{-6}$ Torr. Palladium Schottky contacts of diameter $0.6 \mathrm{~mm}$ and thickness of $60 \mathrm{~nm}$ were fabricated on the O-polar face of the $\mathrm{ZnO}$ samples using the resistive evaporation system under a vacuum of approximately $1 \times 10^{-6}$ 
Torr. Temperature-dependent $I-V$ measurements were performed in a closed cycle Helium cryostat in the $30-300 \mathrm{~K}$ temperature range.

\section{Results and discussion}

Figure 2, shows the semi-logarithmic plot of $I-V$ characteristics of $\mathrm{Pd} / \mathrm{ZnO}$ obtained in the 80-300 K temperature range. The curves obtained from figure 2 , show a very strong

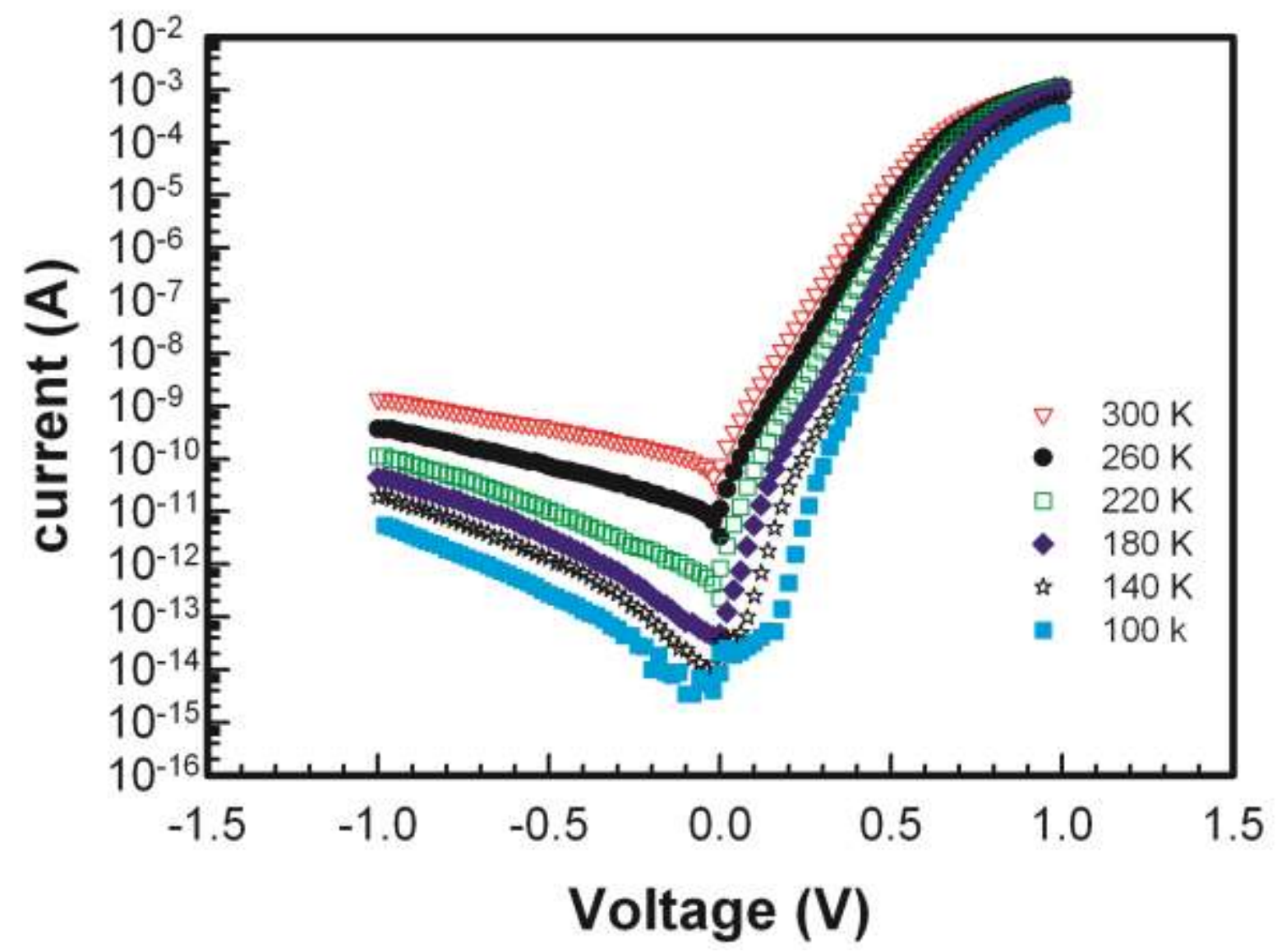

Figure 2. Temperature dependent $I-V$ semi logarithmic plot for the As-deposited $\mathrm{Pd} / \mathrm{ZnO} \mathrm{SBD}$ in the $80-300 \mathrm{~K}$ temperature range.

temperature dependence of the $\mathrm{Pd} / \mathrm{ZnO}$ Schottky barrier diodes. It can be clearly observed that the characteristics of the device deviate from ideality at low temperatures (30-140 K). Such deviations are usually attributed to the effect of other current transport mechanisms, which include the generation-recombination of the carriers in the space charge region and 
tunneling of electrons through the barrier [20]. At temperatures above $200 \mathrm{~K}$, thermionic emission becomes the dominant current transport mechanism [1, 26]. From Figure 2, the experimental values of ideality factor $(n)$ and the barrier height $\left(\Phi_{a p}\right)$ were determined using the intercepts and the gradients of the forward bias $I-V$ characteristics at each temperature, respectively by fitting the pure thermionic emission model to the experimental data.

Using the pure thermionic emission model, the current through a uniform metalsemiconductor interface can be expressed as [27],

$I=I_{S} \exp \left[\frac{q\left(V-I R_{S}\right)}{n k T}\right]\left\{1-\exp \left(\frac{q\left(V-I R_{S}\right)}{k T}\right)\right\}$

Where $R_{S}$ is the series resistance, $q$ is the electron charge, $V$ is the forward-bias voltage, $k$ is the Boltzmann constant, $T$ is the absolute temperature, $n$ is the ideality factor and $I_{S}$ is the saturation current derived from the straight-line intercept of the $\ln I$ versus $V$ i.e. at $(V=0 V)$ and is defined by,

$I_{S}=A A^{*} T^{2} \exp \left(-\frac{q \phi_{a p}}{k T}\right)$

where $A$ is the effective diode area, $A^{*}$ is the effective Richardson constant, $\Phi_{a p}$ is the apparent zero-bias barrier height. The ideality factor $(n)$ in equation 1 can be obtained as[28],

$n=\frac{q}{k T}\left(\frac{d V}{d \ln I}\right)$

The zero-bias barrier height $\Phi_{B o}(I-V)$ is determined from the saturation current $I_{S}$, obtained at $V=0 \mathrm{~V}$ and is given by,

$\phi_{B o}=\frac{k T}{q} \ln \left[\frac{A A^{*} T^{2}}{I_{S}}\right]$

Figure 3, shows a plot of barrier height $\Phi_{a p}$ and the ideality factor as a function of temperature. The zero-bias barrier height values have been calculated from equation 2 by assuming pure thermionic emission of charge carriers across the barrier. The ideality factor decrease with increase in temperatures. The obtained high values at lower temperature can be attributed to the change in the current transport process from generation recombination and 


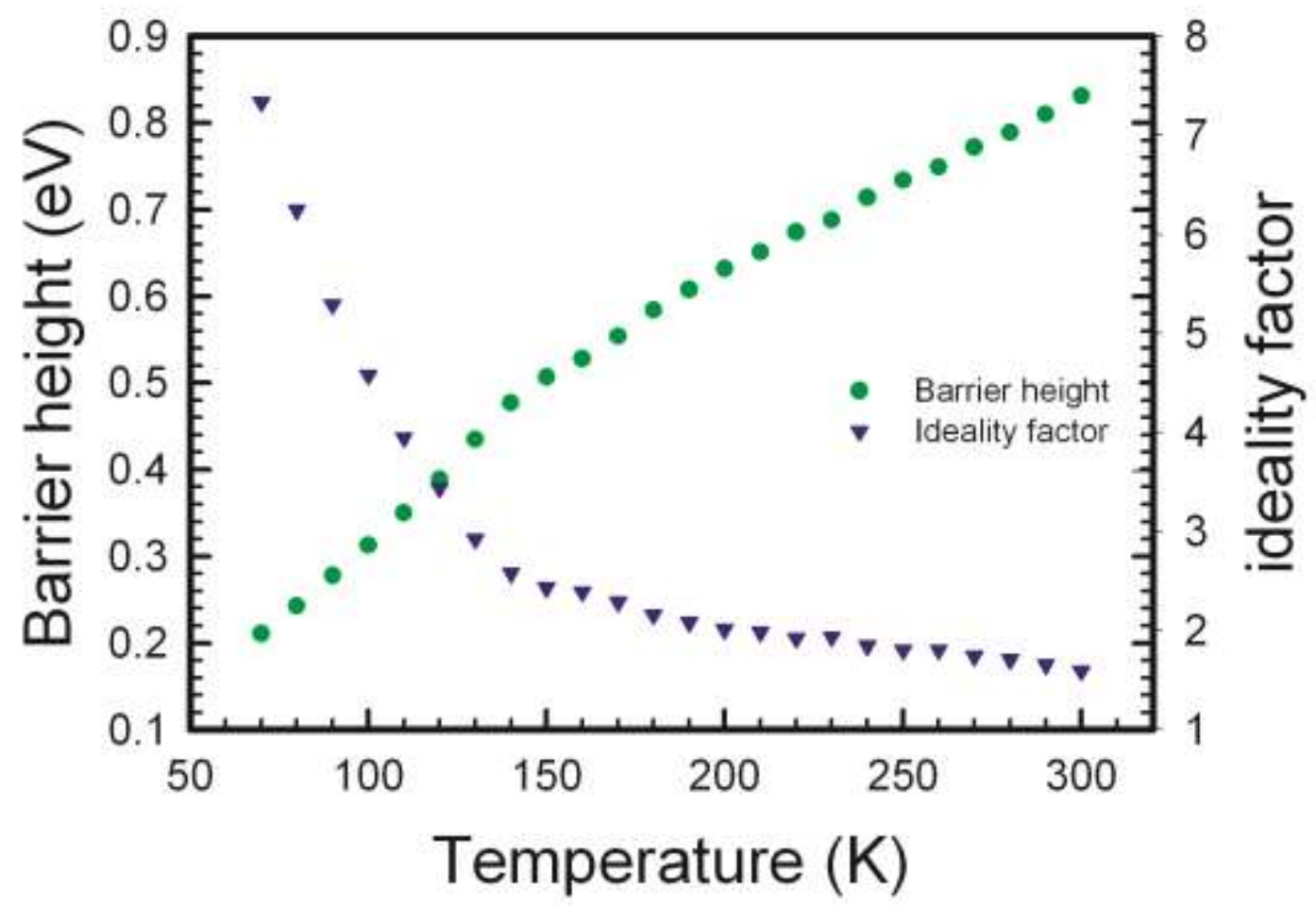

Figure 3. Barrier height and ideality factor as a function of temperature in the $80-300 \mathrm{~K}$ temperature range for $\mathrm{Pd} / \mathrm{ZnO}$ SBD.

other transport mechanisms. A similar trend were also observed by several authors $[3,17,19$, 20]. The observed increase in barrier height with increase in temperature disagrees with the negative temperature coefficient of II-IV semiconductor material [29, 30]. Several researchers have observed the same trend on various Schottky contacts $[12,27,30,31]$ and they have explained it to be possibly related to the temperature-activated current transport of carriers across the MS interface. These carriers at low temperatures are able to surmount the lower barriers through tunneling and therefore the current will be dominated by transport of charge carriers through the patches of lower SBH. As the temperature increases, many electrons gain sufficient energy to surmount the higher barrier areas through thermionic emission $[3,4]$. As a result, the obtained barrier height will increase with increase in temperature and bias voltage. 


\subsection{Richardson constant}

To obtain the mean barrier height and the Richardson constant in a range of temperatures, equation 2, can be linearized as [32],

$\ln \left(\frac{I_{S}}{T^{2}}\right)=\ln \left(A A^{*}\right)-\frac{q \Phi_{B O}}{k T}$

The Richardson constant can also be determined from the intercept of the $\ln \left(I_{S} / T^{2}\right)$ versus $10^{3} / T$ plot shown on Figure 4. The plot shows some linear behavior at high temperatures

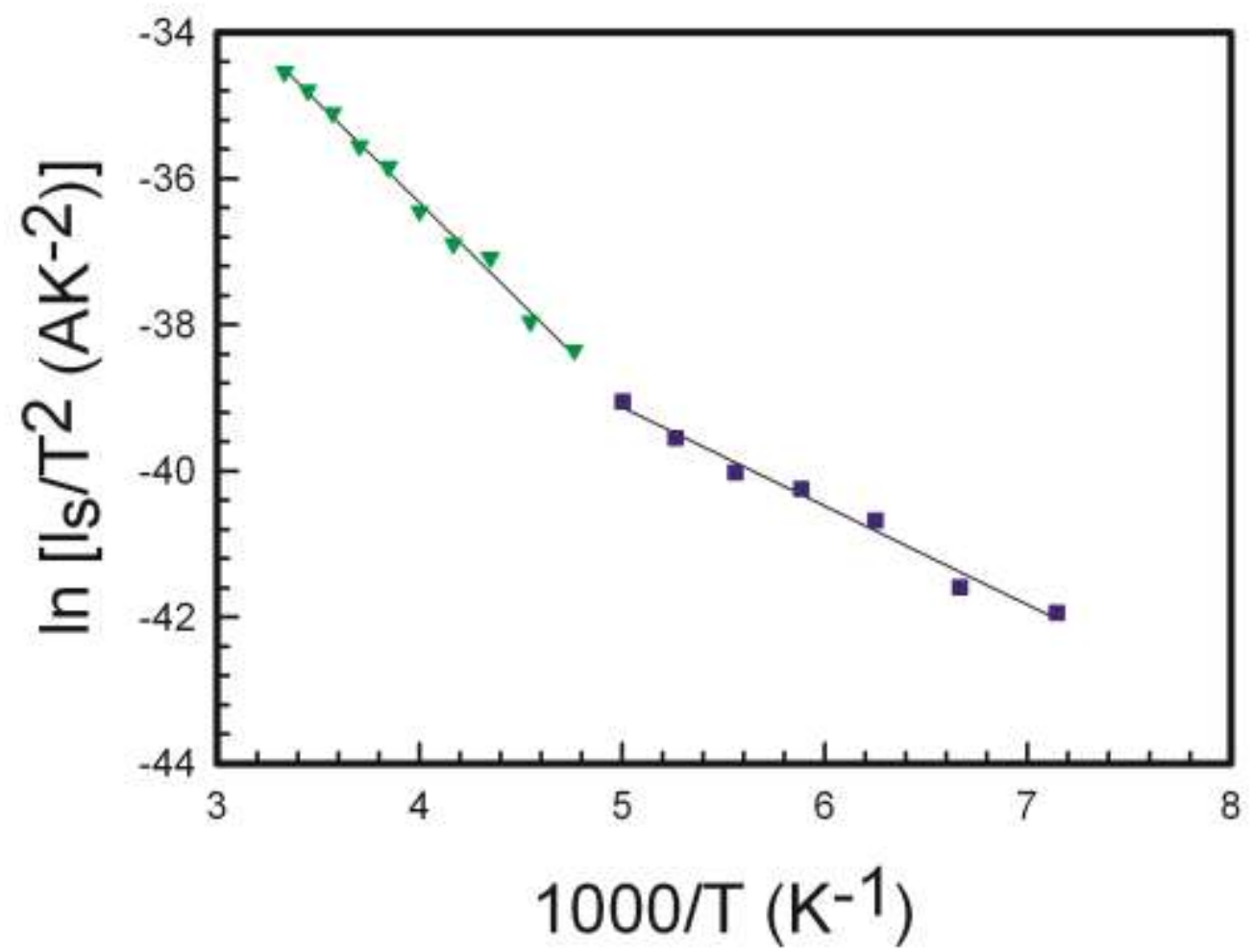

Figure 4. Richardson plot, $\ln \left(I_{s} / T^{2}\right)$ versus $1000 / T$, for the $\mathrm{Pd} / \mathrm{ZnO}$ SDB in the $80-30 O K$ temperature range.

(140-200 and 210-300K) and a deviation from linearity is observed in the temperature range below $140 \mathrm{~K}$. The non-linearity of the conventional energy $\ln \left(I_{S} / T^{2}\right)$ versus $10^{3} / T$ plot is caused by the dependence of the barrier height and the ideality factor on temperature reported by several researchers $[9,14,31,33]$. Two distinct regions have been identified where linear plots could be fitted to the experimental data. From these two regions, the 
Richardson constant values are $3 \times 10^{-12}$ and $3 \times 10^{-9} \mathrm{~A} \mathrm{~cm}^{-2} \mathrm{~K}^{-2}$ in the $140-200 \mathrm{~K}$ and 210-300 K temperature ranges, respectively. These values are much lower compared to the theoretical value of $32 \mathrm{~A} \mathrm{~cm}^{-2} \mathrm{~K}^{-2}$ in $\mathrm{n}-\mathrm{ZnO}$. An effective barrier height of 0.11 and $0.24 \mathrm{eV}$ has been calculated in the $140-200 \mathrm{~K}$ and $210-300 \mathrm{~K}$ temperature range. The deviation in the Richardson plot may be due to the presence of the spatially inhomogeneous barrier height and the potential fluctuations at the interface that consists of low and high barrier areas, i.e. when the temperature is lowered, the current will flow preferentially through the lower barriers in the potential distribution $[9,12,14,20,31]$.

\subsection{Barrier Height inhomogeneities}

In addressing the observed inhomogeneities in the Schottky barrier diodes, which deviates from classical TE theory, some researchers have considered a system of discrete regions of low barrier areas imbedded in a high background of uniform barrier [34-36]. These behaviors can be explained by assuming the Gaussian distribution of the barrier heights with a mean value $\Phi_{b}$ and standard deviation $\sigma_{s}$, which can be given by [37],

$\mathrm{P}\left(\emptyset_{b}\right)=\frac{1}{\sigma_{s} \sqrt{2 \pi}} \exp \left[-\frac{\left(\Phi_{b}\right)-\bar{\Phi}_{b}}{2 \sigma_{s}^{2}}\right]$

Where $1 / \sigma_{s} \sqrt{2 \pi}$ is the normalization constant of the Gaussian barrier height distribution. The total I(V) across a Schottky diode containing a barrier inhomogeneities can be expressed as [34],

$I(V)=\int_{-\infty}^{+\infty} I\left(\Phi_{b}, V\right) P\left(\Phi_{b}\right) d \phi_{b}$

where $I\left(\Phi_{b}, V\right)$ is the current at a bias $V$ for a barrier height based on the ideal thermionic emission-diffusion (TED) theory and $P\left(\Phi_{b}\right)$ is the normalization distribution function giving the probability of accuracy for the barrier height [38]. Substituting equation 1 for $I\left(\Phi_{b}, V\right)$ and equation 6 for $P\left(\Phi_{b}\right)$ in equation 7 , we obtain the current $I(V)$ through the Schottky barrier at a forward bias V but with a modified barrier as [34], 
$\mathrm{I}(\mathrm{V})=\mathrm{I}_{\mathrm{s}} \exp \left(\frac{q V}{n_{a p} k T}\right) \times\left[1-\exp \left(-\frac{q v}{k T}\right)\right]$

with

$I_{s}=A A^{*} T^{2} \exp \left(-\frac{q \Phi_{a p}}{k T}\right)$

where $n_{a p}$ and $\Phi_{a p}$ are the apparent ideality factor and apparent barrier height at zero bias, respectively, given by [24, 28],

$\Phi_{a p}=\Phi_{b}(T=0)-\frac{q \sigma_{s o}^{2}}{2 k T}$

and

$\left(\frac{1}{n_{a p}}-1\right)=\rho_{2}-\frac{q \rho_{3}}{2 k T}$

It is assumed that the mean $\mathrm{SBH}, \Phi_{b}$ and the standard deviation, $\sigma_{s}$ are linearly bias-dependent on Gaussian parameters, such that $\bar{\Phi}_{\mathrm{b}}=\bar{\Phi}_{\mathrm{bo}}+\rho_{2} V$ and a standard deviation $\sigma_{s}=\sigma_{s o}+\rho_{3} V$, where $\bar{\Phi}_{\mathrm{bo}}$ is the barrier height at temperature $T=0 \mathrm{~K}, \rho_{2}$ and $\rho_{3}$ are voltage coefficients which may depend on temperature, quantifying the voltage deformation of the barrier height distribution [20]. The temperature dependence of $\sigma_{s}$ is small and therefore can be neglected. The decrease of zero-bias barrier height is caused by the existence of a Gaussian distribution and the extent of influence is determined by the standard deviation itself $[20,34]$.

The current transport of electrons across a Schottky barrier is affected by the presence of barrier inhomogeneities. These are attributed to fact that charge carriers have insufficient energy to surmount the barriers at low temperatures. Thereby, at these temperature ranges tunneling becomes the dominant transport mechanism of electrons. The tunneling of the electrons through low barrier areas will cause a deviation of the barrier height from the actual value obtained for a homogeneous barrier at the metal-semiconductor interface. A linear fit for the apparent ideality factor, which obeys equation 10 has been obtained and shown in figure 5. In the low temperature region, the decrease in $\Phi_{B o}$ is caused by the existence of the 


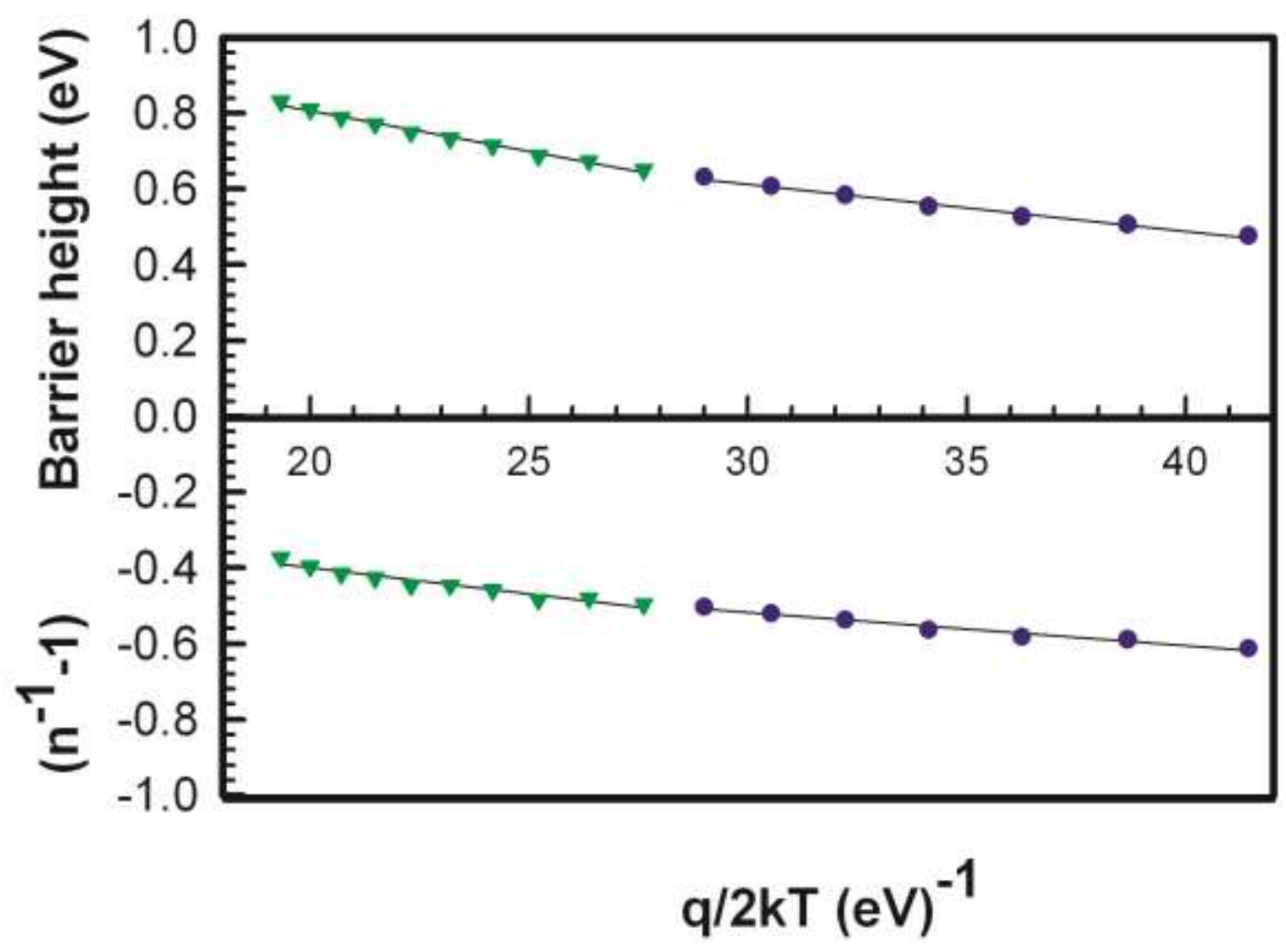

Figure 5. Zero-bias apparent barrier height and ideality factor versus q/2kT curves of the $\mathrm{Pd} / \mathrm{ZnO} \mathrm{SBD}$ according to the Gaussian distribution of the barrier heights.

Gaussian distribution and the extent of influence is determined by the standard deviation itself. In addition, the effect is particularly significant at low temperatures [20]. The fitting of the experimental data using equation 2 or 9 and in equation 3 gives $\Phi_{a p}$ and $n_{a p}$ at zero bias, respectively, which should obey equations 10 and 11 . Thus, the plot of $\Phi_{a p}$ versus $q / 2 k T$ (figure 5) should be a straight line that gives $\Phi_{b o}$ and $\sigma_{s o}$ from the intercepts and the slope, respectively. From figure 4, the values of $\Phi_{b o}$ are 0.986 and $1.234 \mathrm{eV}$ in the $140-200 \mathrm{~K}$ and 210-300 K temperature ranges. Von Wenchstrern et al [21] obtained similar results. The values of $\sigma_{\text {so }}$ are 109 and $144 \mathrm{mV}$ in the $140-200 \mathrm{~K}$ and $210-300 \mathrm{~K}$ temperature ranges, respectively. The lower the value of the $\sigma_{s o}$, the more homogeneous the barrier height is and the 
better the diode rectifying performance [20]. The temperature dependence of the ideality factor can be understood based on equation 11 . Figure 5 , shows a linear fit of the ideality factor where the voltage coefficients $\rho_{2}$ and $\rho_{3}$ are obtained from the intercept and slope, respectively. From figure $5, \rho_{2}=-256$ and $-123 \mathrm{mV}$ and $\rho_{3}=-8.7$ and $-13.9 \mathrm{mV}$ in the $140-200 \mathrm{~K}$ and 210-300 K temperature ranges, respectively. The linear behavior of the plot shows that the ideality factor expresses the voltage deformation of the Gaussian distribution of the SBD.

\subsection{The modified Richardson plot}

As indicated in figure 3 , the saturation current plot, $\ln \left(I_{s} / T^{2}\right)$ versus $10^{3} / T$ has shown non-linearity at low temperatures. To address these observed irregularities, one can correct for the barrier height inhomogeneities according to the Gaussian distribution of the barrier height and can therefore modify equation 5 to give [24, 35],

$I_{s}=A A^{* *} T^{2} \exp \left[-\frac{q \Phi_{a p}}{k T}+\frac{q^{2} \sigma_{S o}^{2}}{2 k^{2} T^{2}}\right]$

and

$\ln \left(\frac{I_{s}}{T^{2}}\right)-\left(\frac{q^{2} \sigma_{s o}^{2}}{2 k^{2} T^{2}}\right)=\ln \left(A A^{* *}\right)-\frac{q \Phi_{a p}}{k T}$

Figure 6, shows a modified Richardson plot, Using the experimental $I_{S}$ data. A modified $\ln \left(I_{s} / T^{2}\right)-\left(q^{2} \sigma_{s o}^{2} / 2 k^{2} T^{2}\right)$ versus $10^{3} / T$ plot according to equation 13 should give a straight line with a slope directly yielding the mean barrier height and the intercept $\ln \left(A A^{* *}\right)$ relating to the modified Richardson constant $A^{* *}$ for a given diode area $A$. The two values of $\sigma_{s o}$ were calculated from figure 5 , in the $140-200 \mathrm{~K}$ and $210-300 \mathrm{~K}$ temperature ranges. From the straight line of the modified saturation current plots, the mean zero-bias barrier heights were obtained as 0.98 and $1.24 \mathrm{eV}$ in the $140-200 \mathrm{~K}$ and $210-300 \mathrm{~K}$ temperature ranges, respectively. The intercepts give $A^{* *}$ as 39.44 and $39.03 \mathrm{~A} \mathrm{~cm}^{-2} \mathrm{~K}^{-2}$ in the $140-200$ and 210-300 K temperature ranges, respectively. These values are in a close agreement with the theoretical value of $32 \mathrm{~A} \mathrm{~cm}^{-2} \mathrm{~K}^{-2}$ compared to those reported in table 1 , by several re 


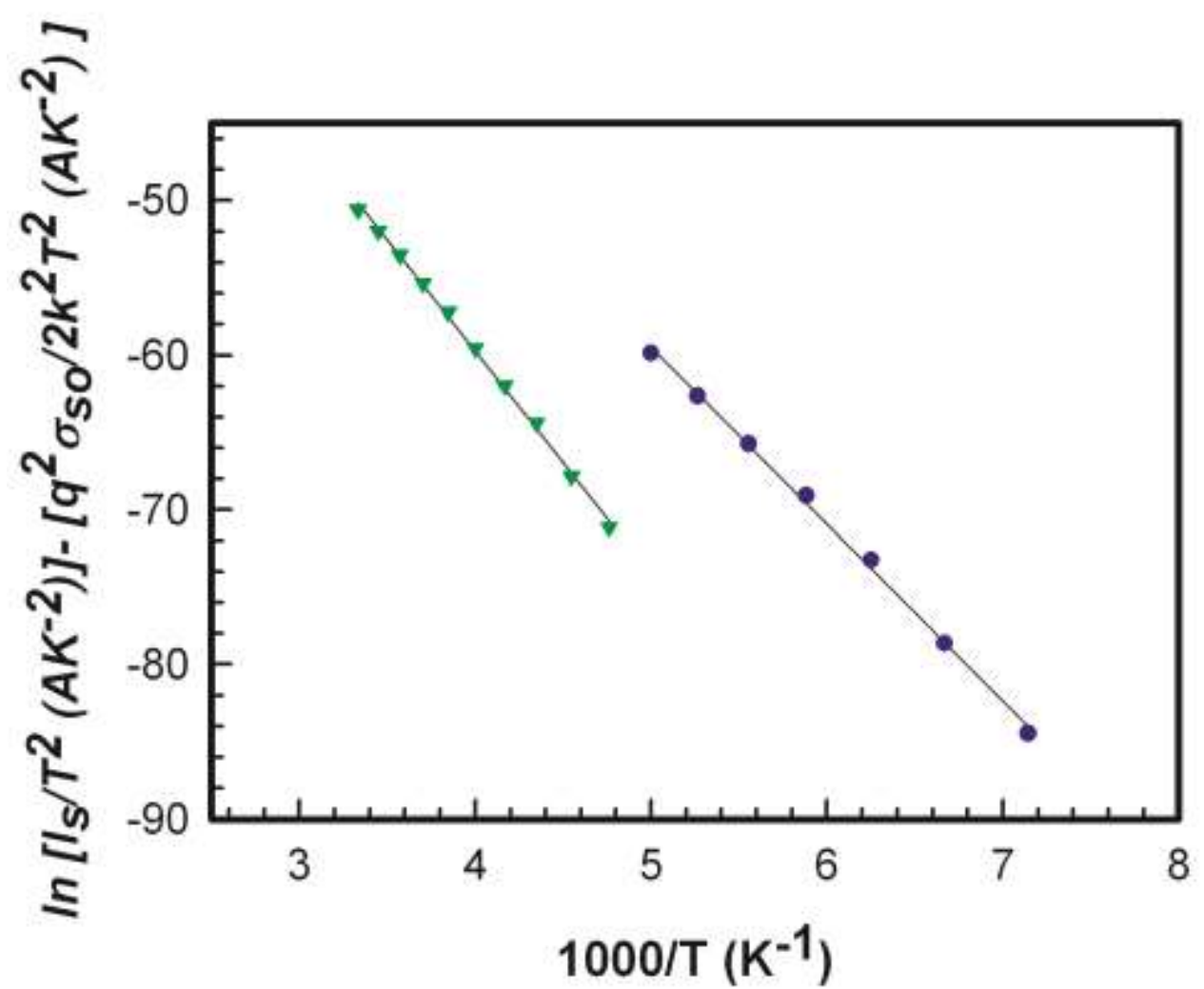

Figure 6. Modified Richardson plot for the Pd/ZnO SBD according to the Gaussian distribution of the barrier heights.

searchers $[2,17,20,22,23]$. The difference in the calculated values of the Richardson constant for different temperature ranges can be attributed to inhomogeneity in the barrier. These findings shows how $I-V$ characteristics can be modeled using TE model at high temperatures indicating that TE is the dominant transport mechanism, while at low temperatures, the abnormal behaviors can be attributed to current flow through the low Schottky barrier areas and tunneling.

\section{Conclusion}

We have investigated the $I-V$ characteristics of $\mathrm{Pd} / \mathrm{ZnO}$ SBDs in the $30-300 \mathrm{~K}$ temperature range. From the temperature dependence of $I-V$ measurements, the $\mathrm{BH}$ was observed to increase with increasing temperature and ideality factor decreases with increasing temperature. The observed variation of the barrier height with temperature disagrees with the negative 
temperature coefficient of II-VI compound semiconductor materials. Apparent Richardson constant, $A^{*}$ values were obtained as $3.03 \times 10^{-12}$ and $3.28 \times 10^{-9} \mathrm{~A} \mathrm{~cm}^{-2} \mathrm{~K}^{-2}$ in the $140-200$ $\mathrm{K}$ and 210-300 $\mathrm{K}$ temperature range, respectively. The values of $A^{*}$ has showed a strong dependence on the metal-semiconductor contact quality and saturation current. After the barrier height inhomogeneities correction, the modified Richardson constant, $A^{* *}$ obtained from the modified Richardson plot, was 39.44 and $39.03 \mathrm{~A} \mathrm{~cm}^{-2} \mathrm{~K}^{-2}$ in the $140-200 \mathrm{~K}$ and $210-300 \mathrm{~K}$ temperature range, respectively. The modified Richardson constant obtained in the modified plot is closer to the theoretical value for $\mathrm{n}-\mathrm{ZnO}$. The mean barrier height was evaluated to be $0.98 \mathrm{eV}$ in the $140-200 \mathrm{~K}$. The value of $A^{* *}$ proved to be strongly affected by barrier inhomogeneities and dependent on contact quality. At high temperatures $(210-300 \mathrm{~K})$, the $I-V$ characteristics can be modeled using the thermionic emission model, an indication that thermionic emission is the dominant current transport mechanism, while at low temperatures, the abnormal behaviors in the $I-V$ curves can be attributed to current flow through the low Schottky barrier areas and tunneling.

\section{Acknowledgement}

This work has been made successful through the financial support of the South African National Research Foundation (NRF) and the University of Pretoria. 


\section{Reference}

[1] S.M. Faraz, V. Khranovskyy, R. Yakimova, A. Ulyashin, Q. Wahab, RSM2011, Kota Kinabalu, Malaysia, (2011).

[2] E. Gür, S. Tüzemen, B. Kiliç, C. Coşkun, Journal of Physics: Condensed Matter (2007), 196-206.

[3] W. Mtangi, F.D. Auret, C. Nyamhere, P. Janse van Rensburg, A. Chawanda, M. Diale, J.M. Nel, W.E. Meyer, Physica B: Condensed Matter, 404 (2009) 4402-4405.

[4] Ü. Özgür, Y.I. Alivov, C. Liu, A. Teke, M.A. Reshchikov, S. Doğan, V. Avrutin, S.-J. Cho, H. Morkoç, Journal of Applied Physics, 98 (2005) 041-301.

[5] H.L. Mosbacker, Y.M. Strzhemechny, B.D. White, P.E. Smith, D.C. Look, D.C. Reynolds, C.W. Litton, L.J. Brillson, Applied Physics Letters, 87 (2005) 012-102.

[6] D.J. Rogers, F. Hosseini Teherani, A. Ougazzaden, S. Gautier, L. Divay, A. Lusson, O. Durand, F. Wyczisk, G. Garry, T. Monteiro, M.R. Correira, M. Peres, A. Neves, D. McGrouther, J.N. Chapman, M. Razeghi, Applied Physics Letters, 91 (2007) 071-120.

[7] W. Mtangi, J.M. Nel, F.D. Auret, A. Chawanda, M. Diale, C. Nyamhere, Physica B: Condensed Matter, 407 (2012) 1624-1627.

[8] M. Allen, M. Alkaisi, S. Durbin, Applied physics letters, 89 (2006) 103520-103520-103523.

[9] I. Afandiyeva, S. Demirezen, Ş. Altındal, Journal of Alloys and Compounds, 552 (2013) 423-429.

[10] I. Hussain, M.Y. Soomro, N. Bano, O. Nur, M. Willander, Journal of Applied Physics, 112 (2012) 064-506.

[11] M. Asghar, K. Mahmood, M. Faisal, M. Hasan, Journal of Physics: Conference Series, IOP Publishing (2013) 012-030.

[12] A. Özdemir, A. Turut, A. Kökçe, Semiconductor science and technology, 21 (2006) 298. 
[13] A.J. Mathai, C.K. Sumesh, B.P. Modi, Physics of semiconductor devices (2011).

[14] H. Korkut, N. Yildirim, A. Turut, Microelectronic Engineering, 86 (2009) 111-116.

[15] S.S. Naik, V.R. Reddy, Advanced Materials Letters, 3 (2012) 188-196.

[16] D.S. Reddy, M.B. Reddy, N.N.K. Reddy, V.R. Reddy, Journal of Modern Physics, 2 (2011) 113 123.

[17] H. Kim, A. Sohn, Y. Cho, D.-W. Kim, Journal of Electronic Packaging, 135 (2013) 011010 011010.

[18] I. Hussain, M. Soomro, N. Bano, O. Nur, M. Willander, Journal of Applied Physics, 113 (2013) 234-509.

[19] H. Asil, K. Çinar, E. Gür, C. Coşkun, S. Tüzemen, International Journal, 8 (2013) 371-379.

[20] W. Mtangi, F.D. Auret, C. Nyamhere, P.J. Janse van Rensburg, C.M.D. A, Physica B: Condensed Matter, 404 (2009) 1092-1096.

[21] H. von Wenckstern, G. Biehne, R.A. Rahman, H. Hochmuth, M. Lorenz, M. Grundmann, Applied physics letters, 88 (2006) 092-102.

[22] D. Somvanshi, S. Jit, Electron Device Letters, IEEE, 34 (2013) 1238-1240.

[23] S. Ranwa, P. Kumar Kulriya, V. Dixit, M. Kumar, Journal of Applied Physics, 115 (2014) 233706-233706-233706.

[24] S. Chand, J. Kumar, Applied Physics A, 63 (1996) 171-178.

[25] http://www.cermetinc.com/materials/n $\quad \mathrm{ZnO}$ substrate product lit(2014).

[26] R. Yatskiv, J. Grym, Applied Physics Letters, 101 (2012) 162-106.

[27] D. Korucu, A. Turut, H. Efeoglu, Physica B: Condensed Matter, 414 (2013) 35-41. 
[28] A. Gümüş, A. Türüt, N. Yalçin, Journal of Applied Physics, 91 (2002) 245-250.

[29] B.G. Yacobi, Semiconductor Materials: An Introduction to Basic Principles, Springer US (2013).

[30] E. Özavc1, S. Demirezen, U. Aydemir, A. Ş, Sens Actuators A Phys, 194 (2013) 259-268.

[31] H. Tecimer, A. Türüt, H. Uslu, Ş. Altındal, İ. Uslu, Sensors and Actuators A: Physical, (2013).

[32] J.H. Werner, H.H. Güttler, Journal of Applied Physics, 69 (1991) 1522-1533.

[33] D. Korucu, S. Duman, Thin Solid Films, (2013) 436-441.

[34] İ. Dökme, Ş. Altindal, M.M. Bülbül, Applied Surface Science, 252 (2006) 7749-7754.

[35] Y. Song, R. Van Meirhaeghe, W. Laflere, F. Cardon, Solid-State Electronics, 29 (1986) 633-638.

[36] S. Chand, Semiconductor Science and Technology, 19 (2004) 82-86.

[37] N. Bannov, V. Aristov, V. Mitin, R.E. Schmitsdorf, T.U. Kampen, W. Monch, R.F. Schmitsdorf, Surface Science, 324 (1995) 249-256.

[38] S. Chand, J. Kumar, Journal of applied physics, 82 (1997) 5005-5010.

[39] M.W. Allen, X. Weng, J.M. Redwing, K. Sarpatwari, S.E. Mohney, H. von Wenckstern, M. Grundmann, S.M. Durbin, Electron Devices, IEEE Transactions on, 56 (2009) 2160-2164. 